\title{
Influence of nonideal LRL or TRL calibration elements on VNA S-parameter measurements
}

\author{
U. Stumper \\ Physikalisch-Technische Bundesanstalt, Bundesallee 100, 38116 Braunschweig, Germany
}

\begin{abstract}
For the 7-term LRL and TRL calibration of a foursampler vector network analyser (VNA), expressions for the deviations of the measured S-parameters of two-port test objects from their actual values are presented as functions of the deviations of the S-parameters of the LRL/TRL calibration elements from their ideal values. The obtained sensitivity coefficients are suitable for establishing the Type-B uncertainty budget for S-parameter measurements. They show how the measurements are affected by imperfect calibration elements and nonideal connections.
\end{abstract}

\section{Introduction}

When applying the frequently used 7-term LRL calibration method to a 4-sampler VNA, its two measuring ports 1 and 2 are connected via a length of an ideally nonreflecting line (a precision air-line impedance standard "Line 1" defining the characteristic impedance), then via a second ideally nonreflecting line "Line 2" (an impedance standard of a length different from that of Line 1 but of the same characteristic impedance) and are then terminated by reflective loads, usually short-circuits R ("Reflect") of ideally equal reflection coefficients. In most cases, the Lines and Reflects are parts of commercial calibration kits. When applying the wide-spread TRL calibration method, one of the Lines is replaced by an ideally nonreflecting "Through" connection with transmission One (i.e. by connecting the measuring ports directly). By means of the VNA firmware, from the raw reflection and transmission values determined for the three calibration states, the seven error terms which characterize the VNA, and additionally the characteristics of the Lines and Reflects ("self-calibration") are calculated. There it is assumed that the calibration elements have the ideal properties as specified.

Correspondence to: U. Stumper

ulrich.stumper@ptb.de
However, the S-parameters associated with the Line 1 (or Through), Line 2, and Reflects as taken from the calibration kit and connected to the measuring ports show deviations from the ideal values assigned to the calibration elements by the VNA firmware. Consequently, the calculated error terms will deviate from their real values which are defined by the VNA hardware, and consequently, the S-parameters of subsequently measured test objects evaluated by use of these incorrect error terms will show deviations $\delta S_{\mathrm{jk}}$ from their actual S-parameters $S_{\mathrm{jk}}$. The aim of this paper, which is an extension of previous work (Stumper, 2004, 2005), is to show how these deviations $\delta S_{\mathrm{jk}}$ depend on the deviations from the ideal S-parameters of the calibration elements, e.g. to calculate the sensitivity coefficients assigned to them. These can be used to establish the Type-B uncertainty budget, according to well known guidelines (GUM, 1995; Guidelines, 2000), where it is necessary to calculate the individual uncertainty contributions associated with the different input estimates.

For the derivation of the deviations, the scattering parameter notation (Rytting, 2001) is used here instead of the usual depiction of the TRL or LRL methods using cascade matrices. Effects on the raw values e.g. caused by noise or nonlinearity and cross-talk effects are not considered here.

\section{LRL calibration method and evaluation of measure- ments}

In the literature, the characteristics of the VNA are most commonly described in terms of cascade matrices of the error terms (e.g. Gronau, 2001). However, for the error analysis depicted here, scattering matrices are used (Rytting, 2001) allowing simplest calculations. For better understanding of the analysis, a short description is given first showing how the error terms are obtained with the LRL calibration method when scattering parameter notation is used.

The actual S-parameters $S_{\mathrm{jk}}$ of calibration elements or of the test object and the non-corrected S-parameters (raw values $m_{\mathrm{jk}}$ ) measured by the VNA are related 
$\mathbf{a}_{\mathbf{0}}$

$\mathbf{b}_{\mathbf{0}}$

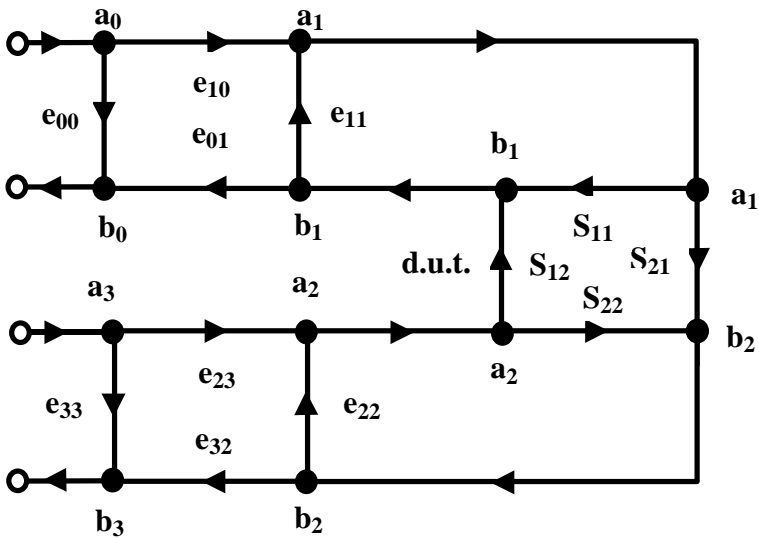

Fig. 1. Flow graph of the 8-term error model (Rytting, 2001). With an LRL calibration, the test object is replaced by the LRL calibration elements (Line 1, Line 2 and Reflective Loads).

to 8 error terms $e_{00}, e_{01}, e_{10}, e_{11}, e_{22}, e_{23}, e_{32}$, and $e_{33}$ (cf. Fig. 1). By normalization, the number of error terms is reduced to seven, and that, for simplification, with respect to Line 1 with an associated transmission coefficient $L_{1}: a=e_{00}, \quad b=L_{1} \cdot e_{11} \cdot\left(e_{23} / e_{10}\right)$, $c=L_{1} \cdot\left(e_{00} e_{11}-e_{10} e_{01}\right) \cdot\left(e_{23} / e_{10}\right), \quad d=L_{1} \cdot e_{22} \cdot\left(e_{10} / e_{23}\right)$, $e=e_{33}, \quad f=L_{1} \cdot\left(e_{22} e_{33}-e_{32} e_{23}\right) \cdot\left(e_{10} / e_{23}\right), \quad g=\left(e_{10} / e_{23}\right)$. With commercial VNAs, the moduli of $c, f$, and $g$ are in the order of 1 while the moduli of $a, b, d$, and $e$ are in the order of 0.1 or less. Using $r_{\mathrm{jk}}=S_{\mathrm{jk}} / L_{1}$, the relations between raw values, $S$-parameters and these 7 error terms are then in "linear-in-T" form (Rytting, 2001)

$a+r_{11} m_{11} b g-r_{11} c g+r_{21} m_{12} d=m_{11}$

$r_{12} m_{11} b g-r_{12} c g+r_{22} m_{12} d-m_{12} g=0$

$r_{11} m_{21} b g+r_{21} m_{22} d-r_{21} f=m_{21}$

$r_{12} m_{21} b g+e g+r_{22} m_{22} d-r_{22} f-m_{22} g=0$

in "measured S-parameters" (raw values) form (Rytting, 2001):

$m_{11}=\frac{\left(a-r_{11} \cdot c g\right) \cdot\left(g-r_{22} \cdot d\right)-r_{12} r_{21} \cdot c d g}{\left(1-r_{11} \cdot b g\right) \cdot\left(g-r_{22} \cdot d\right)-r_{12} r_{21} \cdot b d g}$

$m_{12}=\frac{r_{12} \cdot(a b-c) \cdot g}{\left(1-r_{11} \cdot b g\right) \cdot\left(g-r_{22} \cdot d\right)-r_{12} r_{21} \cdot b d g}$

$m_{21}=\frac{r_{21} \cdot(d e-f) \cdot g}{\left(1-r_{11} \cdot b g\right) \cdot\left(g-r_{22} \cdot d\right)-r_{12} r_{21} \cdot b d g}$

$m_{22}=\frac{\left(1-r_{11} \cdot b g\right) \cdot\left(e g-r_{22} \cdot f\right)-r_{12} r_{21} \cdot b f g}{\left(1-r_{11} \cdot b g\right) \cdot\left(g-r_{22} \cdot d\right)-r_{12} r_{21} \cdot b d g}$.

or in "actual S-parameters" form (Rytting, 2001):

$r_{11}=\frac{\left(m_{11}-a\right) \cdot\left(m_{22} d-f\right)-m_{12} m_{21} d}{\left[\left(m_{11} b-c\right) \cdot\left(m_{22} d-f\right)-m_{12} m_{21} b d\right] \cdot g}$

$$
\begin{aligned}
& r_{12}=\frac{m_{12} \cdot(d e-f)}{\left[\left(m_{11} b-c\right) \cdot\left(m_{22} d-f\right)-m_{12} m_{21} b d\right]} \\
& r_{21}=\frac{m_{21} \cdot(a b-c)}{\left[\left(m_{11} b-c\right) \cdot\left(m_{22} d-f\right)-m_{12} m_{21} b d\right]} \\
& r_{22}=\frac{\left[\left(m_{11} b-c\right) \cdot\left(m_{22}-e\right)-m_{21} m_{12} b\right] \cdot g}{\left[\left(m_{11} b-c\right) \cdot\left(m_{22} d-f\right)-m_{12} m_{21} b d\right]} .
\end{aligned}
$$

For calibration, we connect Line 1 of length $l_{1}$ and propagation coefficient $\gamma$ to the VNA test ports 1 and 2. The undisturbed scattering matrix describing the ideal Line 1 is $\overline{\mathbf{L}_{\mathbf{1}}}=\left(\begin{array}{cc}0 & L_{1} \\ L_{1} & 0\end{array}\right)=\left(\begin{array}{cc}0 & e^{-\gamma l_{1}} \\ e^{-\gamma l_{1}} & 0\end{array}\right)$. By replacing the $r_{\mathrm{jk}}$ by $S_{\mathrm{jk}} / L_{1}$ in Eqs. (1) to (4) and inserting for the $S_{\mathrm{jk}}$ the entries of $\overline{\mathbf{L}_{\mathbf{1}}}$ (Index $\mathrm{T}$ of raw values for Line 1), we obtain:

$a+m_{12}^{T} d=m_{11}^{T}$

$m_{11}^{T} b-c=m_{12}^{T}$

$m_{22}^{T} d-f=m_{21}^{T}$

$m_{21}^{T} b+e=m_{22}^{T}$.

With the connection of an ideal Line 2 of length $l_{2}$, represented by the undisturbed scattering matrix $\overline{\mathbf{L}_{2}}=\left(\begin{array}{cc}0 & L_{2} \\ L_{2} & 0\end{array}\right)=\left(\begin{array}{cc}0 & e^{-\gamma l_{2}} \\ e^{-\gamma} l_{2} & 0\end{array}\right), \quad$ to the test ports, we obtain, by replacing the $r_{\mathrm{jk}}$ by $S_{\mathrm{jk}} / L_{1}$ in Eqs. (1) to (4) and inserting for the $S_{\mathrm{jk}}$ the entries of $\overline{\mathbf{L}_{2}}$ (Index $\mathrm{L}$ of raw values for Line 2):

$a+M m_{12}^{L} d=m_{11}^{L}$

$M m_{11}^{L} b-M c=m_{12}^{L}$

$M m_{22}^{L} d-M f=m_{21}^{L}$

$M m_{21}^{L} b+e=m_{22}^{L}$

where,

$M=L_{2} / L_{1}=\exp \left[-\gamma \cdot\left(l_{2}-l_{1}\right)\right]=\exp (-\gamma \cdot \Delta l)$

If we connect two ideal (i.e. equally reflecting) loads (usually short-circuits), represented by the undisturbed scattering matrix $\overline{\mathbf{R}}=\left(\begin{array}{cc}\Gamma & 0 \\ 0 & \Gamma\end{array}\right)$, to the test ports, we obtain, by replacing the $r_{\mathrm{jk}}$ by $S_{\mathrm{jk}} / L_{1}$ in Eqs. (1) and (4) and inserting for the $S_{\mathrm{jk}}$ the entries of $\overline{\mathbf{R}}$ (Index R for Reflect):

$a+\left(\Gamma / L_{1}\right) \cdot m_{11}^{R} b g-\left(\Gamma / L_{1}\right) \cdot c g=m_{11}^{R}$

$e g+\left(\Gamma / L_{1}\right) \cdot m_{22}^{R} d-\left(\Gamma / L_{1}\right) \cdot f=g \cdot m_{22}^{R}$,

where $S_{12}=S_{21}=m_{12}^{R}=m_{21}^{R}=0$, as cross-talk effects are not considered here.

The error term $g$ is obtained by elimination of $\Gamma / L_{1}$ from Eqs. (22) and (23):

$g^{2}=\left(m_{11}^{R}-a\right) \cdot\left(m_{22}^{R} d-f\right) /\left\{\left(m_{22}^{R}-e\right) \cdot\left(m_{11}^{R} b-c\right)\right\}$.

We obtain 10 equations for 9 unknowns (7 error terms plus $M$ and $\Gamma$ ). The calculation is straightforward. With the "self-calibrating" LRL method, $M$ is determined from the 
raw values obtained for Line 1 and Line 2: By elimination of $f$ from Eqs. (15) and (19), of $a$ from Eqs. (13) and (17), and of $e$ from Eqs. (16) and (20) we obtain

$d=\left(m_{21}^{L}-M m_{21}^{T}\right) /\left(M m_{22}^{L}-M m_{22}^{T}\right)$

$d=\left(m_{11}^{L}-m_{11}^{T}\right) /\left(M m_{12}^{L}-m_{12}^{T}\right)$

$b=\left(m_{22}^{L}-m_{22}^{T}\right) /\left(M m_{21}^{L}-m_{21}^{T}\right)$.

Elimination of $d$ from Eqs. (25) and (26) yields a quadratic equation to be solved for $M$,

$M^{2} \cdot m_{21}^{T} m_{12}^{L}+M \cdot\left\{\left(m_{11}^{L}-m_{11}^{T}\right)\right.$

$\left.\cdot\left(m_{22}^{L}-m_{22}^{T}\right)-m_{12}^{L} m_{21}^{L}-m_{21}^{T} m_{12}^{T}\right\}+m_{12}^{T} m_{21}^{L}=0$.

A second equivalent quadratic equation can be found by other combination of Eqs. (13) to (20) yielding also the relation $m_{12}^{T} m_{21}^{L}=m_{21}^{T} m_{12}^{L}$ (Engen and Hoer, 1979). A physically significant solution will be nearest to $M_{\text {calc. }} \approx \exp (-j \cdot \beta \cdot \Delta l)$ calculated from phase coefficient $\beta$ and mechanically measured $\Delta l$. With known $M$, the terms $b$ and $d$ can now be calculated from Eqs. (25) to (27), then the error terms $a, c$, $e$, and $f$ from Eqs. (13) to (16) or (17) to (20), and finally $g$ from Eqn. (24). All error terms are then known. Measurements can now be evaluated by use of Eqs. (9) to (12) and $r_{\mathrm{jk}}=S_{\mathrm{jk}} / L_{1}$. The $\mathrm{S}$-parameters of test objects are known except for the transmission coefficient $L_{1}$, which has to be separately determined using the known $M$ and the lengths $l_{1}$ and $l_{2}$ of both Lines determined by mechanical measurements:

$-\ln (M)=-\ln \left(L_{2} / L_{1}\right)=\gamma \cdot\left(l_{2}-l_{1}\right)$

$L_{1}=e^{-\gamma \cdot l_{1}}=\exp \left[l_{1} \cdot \ln (M) /\left(l_{2}-l_{1}\right)\right]$.

For the TRL calibration, $L_{1}=1$ and $M_{\mathrm{TRL}}=L_{2}$.

\section{Calculation of the $\delta S_{\mathrm{jk}}$}

As we are interested, in a first step, in the effect of the seven deviations $\delta a, \delta b, \delta c, \delta d, \delta e, \delta f, \delta g$ of the error terms on the deviations $\delta S_{\mathrm{jk}}$ of the measured S-parameters $S_{\mathrm{jk}}$ of a test object, we assume that they are not influenced by $m_{\mathrm{jk}}$ variations.

We use Eqs. (5) to (8) to find at first the dependence of the $\delta r_{\mathrm{jk}}=\delta\left(S_{\mathrm{jk}} / L_{1}\right)$ on the deviations of the error terms. Four total differentials are established which form four linear equations, similarly as in (Stumper, 2003a).

$0=\delta m_{\mathrm{jk}}=\frac{\partial m_{\mathrm{jk}}}{\partial r_{11}} \cdot \delta r_{11}+\frac{\partial m_{\mathrm{jk}}}{\partial r_{12}} \cdot \delta r_{12}+\frac{\partial m_{\mathrm{jk}}}{\partial r_{21}} \cdot \delta r_{21}+\frac{\partial m_{\mathrm{jk}}}{\partial r_{22}} \cdot \delta r_{22}+\frac{\partial m_{\mathrm{jk}}}{\partial a} \cdot \delta a+\frac{\partial m_{\mathrm{jk}}}{\partial b} \cdot \delta b+\frac{\partial m_{\mathrm{jk}}}{\partial c} \cdot \delta c+\frac{\partial m_{\mathrm{jk}}}{\partial d} \cdot \delta d+\frac{\partial m_{\mathrm{jk}}}{\partial e} \cdot \delta e+\frac{\partial m_{\mathrm{jk}}}{\partial f} \cdot \delta f+\frac{\partial m_{\mathrm{jk}}}{\partial g} \cdot \delta g$

(where the indices $\mathrm{jk}$ are $11,12,21$, or 22). After having carried out the differentiations and some rearranging, we finally obtain the following expressions as solutions for the deviations $\delta r_{\mathrm{jk}}$ of a test object:

$$
\begin{aligned}
& \delta r_{11}=\left\{-\frac{\left(1-r_{11} \cdot b g\right)}{g \cdot(a b-c)} \cdot \delta a-\frac{r_{11} \cdot\left(a-r_{11} \cdot c g\right)}{(a b-c)} \cdot \delta b+\frac{r_{11} \cdot\left(1-r_{11} \cdot b g\right)}{(a b-c)} \cdot \delta c+\frac{r_{12} r_{21} \cdot f}{g \cdot(d e-f)} \cdot \delta d-\frac{r_{12} r_{21} \cdot d}{g \cdot(d e-f)} \cdot \delta f-\frac{r_{11}}{g} \cdot \delta g\right\} \\
& \delta r_{12}=\left\{-\frac{r_{12} \cdot\left(a-r_{11} \cdot c g\right)}{(a b-c)} \cdot \delta b+\frac{r_{12} \cdot\left(1-r_{11} \cdot b g\right)}{(a b-c)} \cdot \delta c+\frac{r_{22} r_{12} \cdot f}{g \cdot(d e-f)} \cdot \delta d+\frac{r_{12} \cdot d}{(d e-f)} \cdot \delta e-\frac{r_{22} r_{12} \cdot d}{g \cdot(d e-f)} \cdot \delta f\right\} \\
& \delta r_{21}=\left\{\frac{r_{21} \cdot b}{(a b-c)} \cdot \delta a+\frac{r_{11} r_{21} \cdot c g}{(a b-c)} \cdot \delta b-\frac{r_{11} r_{21} \cdot b g}{(a b-c)} \cdot \delta c-\frac{r_{21} \cdot\left(e g-r_{22} \cdot f\right)}{g \cdot(d e-f)} \cdot \delta d+\frac{r_{21} \cdot\left(g-r_{22} \cdot d\right)}{g \cdot(d e-f)} \cdot \delta f\right\} \\
& \delta r_{22}=\left\{\frac{r_{12} r_{21} \cdot c g}{(a b-c)} \cdot \delta b-\frac{r_{12} r_{21} \cdot b g}{(a b-c)} \cdot \delta c-\frac{r_{22} \cdot\left(e g-r_{22} \cdot f\right)}{g \cdot(d e-f)} \cdot \delta d-\frac{\left(g-r_{22} \cdot d\right)}{(d e-f)} \cdot \delta e+\frac{r_{22} \cdot\left(g-r_{22} \cdot d\right)}{g \cdot(d e-f)} \cdot \delta f+\frac{r_{22}}{g} \cdot \delta g\right\} .
\end{aligned}
$$

As $\delta r_{\mathrm{jk}}=\delta\left(S_{\mathrm{jk}} / L_{1}\right)=\frac{\delta S_{\mathrm{jk}}}{L_{1}}-S_{\mathrm{jk}} \cdot \frac{\delta L_{1}}{L_{1}^{2}}$, the deviations $\delta S_{\mathrm{jk}}$ of the measured S-parameters $S_{\mathrm{jk}}$ of a test object are dependent also on the deviations $\delta M, \delta l_{1}$, and $\delta l_{2}$ of $M$ and the lengths $l_{1}$ and $l_{2}$ :

$\left.\delta S_{\mathrm{jk}}=L_{1} \cdot \delta r_{\mathrm{jk}}+S_{\mathrm{jk}} \cdot \frac{\delta L_{1}}{L_{1}}=L_{1} \cdot \delta r_{\mathrm{jk}}+S_{\mathrm{jk}} \cdot\left[\frac{l_{1}}{\left(l_{2}-l_{1}\right)} \cdot \frac{\delta M}{M}+\ln (M) \cdot \frac{\left(l_{2} \cdot \delta l_{1}-l_{1} \cdot \delta l_{2}\right)}{\left(l_{2}-l_{1}\right)^{2}}\right]\right)$.

For the TRL calibration, $L_{1}=1$ and $S_{\mathrm{jk}}=r_{\mathrm{jk}}, \delta S_{\mathrm{jk}}=\delta r_{\mathrm{jk}}$. 


\section{Calculation of the deviations of the error terms}

\subsection{Nonideal calibration elements}

In a second step, the deviations $\delta a, \delta b, \delta c, \delta d, \delta e, \delta f, \delta g$ are obtained as functions of the deviations from the ideal Sparameters associated with Line 1, Line 2, and Reflect. It is not possible here to use the method shown in (Stumper, 2003a).

The deviations with Line 1, Line 2, and Reflect are described below by the "disturbed" scattering matrices, which are compiled together with the "undisturbed ideal" matrices in Table 1.

\subsection{Determination of the deviation $\delta M$}

At first, $\delta \mathrm{M}$ is determined. By means of the VNA firmware, $M=L_{2} / L_{1}$ is calculated from the raw values obtained for Line 1 and Line 2, thereby ideal scattering matrices $\overline{\mathbf{L}_{\mathbf{1}}}$ and $\overline{\mathbf{L}_{2}}$ are assumed. To calculate a disturbed $M_{g}=M+\delta M$, we replace the $r_{\mathrm{jk}}$ by $S_{\mathrm{jk}} / L_{1}$ in the Eqs. (5) to (8) and insert the $\mathrm{S}$-parameters of the disturbed $\overline{\mathbf{L}_{1}} d$ or $\overline{\mathbf{L}_{2}} d$ (Table 1), respectively, for $S_{11}, S_{12}, S_{21}$, and $S_{22}$. We then calculate disturbed (index g) raw values $m_{\mathrm{jkg}}^{T}, m_{\mathrm{jkg}}^{L}$ which differ from the undisturbed ideal raw values $m_{\mathrm{jk}}^{T}, m_{\mathrm{jk}}^{L}$ by deviations $\delta m_{\mathrm{jk}}^{T}, \delta m_{\mathrm{jk}}^{L}$, respectively. The equation for the disturbed $M_{g}$ is obtained by expanding Eq. (28) by the $\delta$-elements:

$$
\begin{aligned}
& (M+\delta M)^{2} \cdot\left(m_{21}^{T}+\delta m_{21}^{T}\right) \cdot\left(m_{12}^{L}+\delta m_{12}^{L}\right) \\
& +(M+\delta M) \cdot\left\{\begin{array}{c}
{\left[\left(m_{11}^{L}-m_{11}^{T}\right)+\delta m_{11}^{L}-\delta m_{11}^{T}\right] \cdot\left[\left(m_{22}^{L}-m_{22}^{T}\right)+\delta m_{22}^{L}-\delta m_{22}^{T}\right]} \\
-\left(m_{12}^{L}+\delta m_{12}^{L}\right) \cdot\left(m_{21}^{L}+\delta m_{21}^{L}\right)-\left(m_{21}^{T}+\delta m_{21}^{T}\right) \cdot\left(m_{12}^{T}+\delta m_{12}^{T}\right)
\end{array}\right\}+\left(m_{12}^{T}+\delta m_{12}^{T}\right) \cdot\left(m_{21}^{L}+\delta m_{21}^{L}\right)=0
\end{aligned}
$$

If we insert the calculated undisturbed ideal raw values $m_{\mathrm{jk}}^{T}, m_{\mathrm{jk}}^{L}$ and deviations $\delta m_{\mathrm{jk}}^{T}, \delta m_{\mathrm{jk}}^{L}$ into Eq. (37), we finally obtain a rather simple expression for the deviation $\delta M$

$\delta M=\frac{1}{L_{1}} \cdot \delta s_{21}-\frac{M}{L_{1}} \cdot \delta T_{21}$.

\subsection{Deviations of the error terms due to nonideal Reflect}

By means of the VNA firmware, the seven error terms are calculated from raw values obtained for the Reflects, thereby an ideal scattering matrix $\overline{\mathbf{R}}$ is assumed. To obtain disturbed error terms (ideal plus deviation), we replace the $r_{\mathrm{jk}}$ by $S_{\mathrm{jk}} / L_{1}$ in the Eqs. (5) to (8) and insert the S-parameters of the disturbed $\overline{\mathbf{R}_{d}}$ (Table 1), for $S_{11}$ and $S_{22}$. We then calculate disturbed (index $\mathrm{g}$ ) raw values $m_{\mathrm{jjg}}^{R}$ which differ from the undisturbed ideal raw values $m_{\mathrm{jj}}^{R}$ by deviations $\delta m_{\mathrm{jj}}^{R}$.

By a nonideal Reflect, the error terms $a, b, c, d, e$, and $f$ are not affected. Only $g$ is affected here. The equation describing the disturbed $g_{\mathrm{g}}$ is according to Eqn. (24):

$$
\begin{aligned}
g_{\mathrm{g}}^{2} & =\left(g+\delta g_{\mathrm{R}}\right)^{2} \\
& =\frac{\left(m_{11}^{R}+\delta m_{11}^{R}-a\right) \cdot\left\{\left(m_{22}^{R}+\delta m_{22}^{R}\right) \cdot d-f\right\}}{\left(m_{22}^{R}+\delta m_{22}^{R}-e\right) \cdot\left\{\left(m_{11}^{R}+\delta m_{11}^{R}\right) \cdot b-c\right\}} .
\end{aligned}
$$

If we insert the calculated undisturbed ideal raw values $m_{\mathrm{jj}}^{R}$ and deviations $\delta m_{\mathrm{jj}}^{R}$ into Eqn. (39), we finally obtain a simple relation for the deviation $\delta g$

$\delta g=\delta g_{\mathrm{R}}=\frac{g}{2 \cdot \Gamma} \cdot\left(\delta S_{11}^{R}-\delta S_{22}^{R}\right)$

due to a nonideal Reflect.
4.4 Deviations of the error terms due to nonideal Line 1 and Line 2

By a nonideal Line 1, all error terms are affected. The equations describing the disturbed (Index g) error terms $a_{\mathrm{g}}=a+\delta a_{\mathrm{L} 1}, \ldots ., g_{\mathrm{g}}=g+\delta g_{\mathrm{L} 1}$ affected by the S-parameters of a nonideal Line 1 are calculated according to Eqs. (13) to (16) and (25) to (27), for example for $b$ :

$$
b+\delta b_{\mathrm{L} 1}=\frac{m_{22}^{L}-m_{22}^{T}-\delta m_{22}^{T}}{\left(M+\delta M_{\mathrm{L} 1}\right) \cdot m_{21}^{L}-m_{21}^{T}-\delta m_{21}^{T}} .
$$

If we insert the calculated $\delta M_{\mathrm{L} 1}=-\frac{M}{L_{1}} \cdot \delta T_{21}$ and the undisturbed ideal raw values $m_{\mathrm{jk}}^{T}, m_{\mathrm{jk}}^{L}$ and deviations $\delta m_{\mathrm{jk}}^{T}$ into these equations, we finally obtain the error term deviations due to a nonideal Line 1, e.g. for $b$ :

$$
\begin{aligned}
& \delta b_{\mathrm{L} 1}=\frac{\left(1-M^{2} \cdot b d\right) \cdot b}{\left(1-M^{2}\right) \cdot L_{1}} \cdot \delta T_{12} \\
& -\frac{b \cdot M^{2} \cdot(1-b d)}{\left(1-M^{2}\right) \cdot L_{1}} \cdot \delta T_{21}+\frac{\left(1-M^{2} \cdot b d\right)}{\left(1-M^{2}\right) \cdot L_{1} \cdot g} \cdot \delta T_{22} .
\end{aligned}
$$

By a nonideal Line 2, all error terms are affected as well. The equations describing the disturbed error terms $a_{r m g}=a+\delta a_{\mathrm{L} 2}, \ldots . \quad, g_{\mathrm{g}}=g+\delta g_{\mathrm{L} 2}$ affected by the Sparameters of a nonideal Line 2 are calculated according to Eqs. (13) to (16) and (25) to (27), for example for $b$ :

$$
b+\delta b_{\mathrm{L} 2}=\frac{m_{22}^{L}-m_{22}^{T}+\delta m_{22}^{L}}{\left(M+\delta M_{\mathrm{L} 2}\right) \cdot\left(m_{21}^{L}+\delta m_{21}^{L}\right)-m_{21}^{T}} .
$$

If we insert the calculated $\delta M_{\mathrm{L} 2}=\frac{1}{L_{1}} \cdot \delta s_{21}$ and the undisturbed ideal raw values $m_{\mathrm{jk}}^{T}, m_{\mathrm{jk}}^{L}$ and deviations $\delta m_{\mathrm{jk}}^{L}$ into these equations, we finally obtain the error term deviations 
due to a nonideal Line 2, e.g. for $b$ :

$\delta b_{\mathrm{L} 2}=-\frac{M \cdot(1-b d) \cdot b}{L_{1} \cdot\left(1-M^{2}\right)} \cdot \delta s_{12}$
$+\frac{M \cdot(1-b d) \cdot b}{L_{1} \cdot\left(1-M^{2}\right)} \cdot \delta s_{21}-\frac{(1-b d)}{L_{1} \cdot\left(1-M^{2}\right) \cdot g} \cdot \delta s_{22}$.

\section{Resulting sensitivity coefficients}

Combining the results from the first and second step, we obtain sensitivity coefficients for the four S-parameters of a test object which are separately given for deviations with Line 1,
Line 2, and Reflect. If the reflection coefficient of one of the Reflect terminations is $\Gamma$ while the other has a deviating reflection $\Gamma+\delta \Gamma$, there is an influence only on the reflection coefficients, not on the transmission coefficients of test objects, without any influence of $M$ and of any error term. By inserting Eqn. (40) for Reflect into Eqs. (32) and (35), we obtain:

$\left.\delta r_{11}\right|_{\mathrm{R} 1}=-\left.\delta r_{11}\right|_{\mathrm{R} 2}=-\frac{r_{11}}{2 \cdot \Gamma} \cdot \delta \Gamma$

depending on the position of the deviating termination either at port $1\left(\right.$ Index $\left.\mathrm{R} 1: \delta \Gamma=\delta S_{11}^{R}\right)$ or port $2\left(\operatorname{Index} \mathrm{R} 2: \delta \Gamma=\delta S_{22}^{R}\right)$, respectively.

Inserting the deviations of the error terms (cf. Sect. 4.4) obtained for Line 1 into Eqs. (32) to (35), and with $b g=e_{11} \cdot L_{1}$, we obtain (Index L1):

$\delta r_{11 \mathrm{~L} 1}=\left\{\begin{array}{l}\frac{\left(M^{2}-r_{12} r_{21}\right)}{\left(1-M^{2}\right) \cdot L_{1}} \cdot \delta T_{11}-\frac{r_{11} \cdot\left(\Gamma^{2}+L_{1}^{2} \cdot M^{2}\right)}{2 \cdot L_{1}^{2} \cdot\left(1-M^{2}\right) \cdot \Gamma} \cdot\left(\delta T_{11}-\delta T_{22}\right)-\frac{r_{11}}{2 \cdot L_{1}} \cdot\left(\delta T_{12}+\delta T_{21}\right) \\ +\frac{M^{2} \cdot e_{11}}{\left(1-M^{2}\right)} \cdot\left[\frac{r_{11} \cdot\left(\Gamma^{2}+L_{1}^{2}\right)}{2 \cdot L_{1} \cdot \Gamma}-r_{11}^{2}\right] \cdot\left(\delta T_{12}-\delta T_{21}\right)-\frac{r_{11}^{2}}{L_{1} \cdot\left(1-M^{2}\right)} \cdot \delta T_{22}\end{array}\right\}$
$\frac{\delta r_{12 \mathrm{~L} 1}}{r_{12}}=-\frac{r_{22}}{L_{1} \cdot\left(1-M^{2}\right)} \cdot \delta T_{11}-\frac{1}{L_{1}} \cdot \delta T_{12}-\frac{r_{11} \cdot M^{2} \cdot e_{11}}{\left(1-M^{2}\right)} \cdot\left(\delta T_{12}-\delta T_{21}\right)-\frac{r_{11}}{L_{1} \cdot\left(1-M^{2}\right)} \cdot \delta T_{22}$.

Inserting the deviations of the error terms (cf. Sect. 4.4) obtained for Line 2 into Eqs. (32) to (35), and with $b g=e_{11} \cdot L_{1}$, we obtain (Index L2):

$\delta r_{11 \mathrm{~L} 2}=\left\{\begin{array}{l}-\frac{\left(1-r_{12} r_{21}\right)}{L_{1} \cdot\left(1-M^{2}\right)} \cdot \delta s_{11}+\frac{r_{11} \cdot\left(\Gamma^{2}+L_{1}^{2}\right)}{2 \cdot L_{1}^{2} \cdot\left(1-M^{2}\right) \cdot \Gamma} \cdot\left(\delta s_{11}-\delta s_{22}\right) \\ -\frac{M \cdot e_{11}}{\left(1-M^{2}\right)} \cdot\left[\frac{r_{11} \cdot\left(\Gamma^{2}+L_{1}^{2}\right)}{2 \cdot L_{1} \cdot \Gamma}-r_{11}^{2}\right] \cdot\left(\delta s_{12}-\delta s_{21}\right)+\frac{r_{11}^{2}}{L_{1} \cdot\left(1-M^{2}\right)} \cdot \delta s_{22}\end{array}\right\}$

$\frac{\delta r_{12 \mathrm{~L} 2}}{r_{12}}=\frac{\delta r_{21 \mathrm{~L} 2}}{r_{21}}=\frac{r_{22}}{L_{1} \cdot\left(1-M^{2}\right)} \cdot \delta s_{11}+\frac{r_{11} \cdot M \cdot e_{11}}{\left(1-M^{2}\right)} \cdot\left(\delta s_{12}-\delta s_{21}\right)+\frac{r_{11}}{L_{1} \cdot\left(1-M^{2}\right)} \cdot \delta s_{22}$.

For Line 1 and Line 2 , only $e_{11}$ occurs in the sensitivity coefficients. To obtain corresponding expressions for $\delta r_{21}$ and $\delta r_{22}$, index 1 is replaced by 2 and vice versa in Eqs. (45) to (48).

An exchange of Line 1 and Line 2 is based on definitions of an $r_{\mathrm{jk}}^{\prime}=S_{\mathrm{jk}} / L_{2}$ and of an $M^{\prime}=L_{1} / L_{2}$. Then, Eqs. (46) to (49) remain valid, but the $r_{\mathrm{jk}}$ are replaced by $r_{\mathrm{jk}}^{\prime}=S_{\mathrm{jk}} / L_{2}$, and the $\delta s_{\mathrm{ik}}$ are replaced by $\delta T_{\mathrm{jk}}$ and vice versa.

\section{Sensitivity coefficients for the TRL calibration}

If the length of Line 1 diminishes to zero, Eqs. (46) to (49) are valid where $L_{1}=1$ (Through), $r_{\mathrm{jk}}=S_{\mathrm{jk}}, \delta r_{\mathrm{jk}}=\delta S_{\mathrm{jk}}$, and $M=M_{\mathrm{TRL}}=L_{2}=L$ (Line). For nonideal Reflects we then have:

$\left.\delta S_{11}\right|_{\mathrm{R} 1}=-\left.\delta S_{11}\right|_{\mathrm{R} 2}=-\frac{S_{11}}{2 \cdot \Gamma} \cdot \delta \Gamma$.

Experimental verification of Eqs. (46) to (49) showed that, as $e_{11}$ is small, the terms containing $e_{11}$ in the sensitivity coefficients for Through and Line are also small compared with the other terms, so that simplified sensitivity coefficients can be set up, if $|\Gamma|$ is near to One. If we assume reflection symmetry for the nonideal Through and Line and not too large reflections $S_{11}, S_{22}$ of the test objects, the sensitivity coefficients are then approximately given by:

$$
\begin{aligned}
& \delta S_{11 \mathrm{~T}} \approx \frac{\left(L^{2}-S_{12} S_{21}\right)}{\left(1-L^{2}\right)} \cdot \delta T_{11}-\frac{S_{11}}{2} \cdot\left(\delta T_{12}+\delta T_{21}\right) \\
& \frac{\delta S_{12 \mathrm{~T}}}{S_{12}} \approx-\frac{S_{22}}{\left(1-L^{2}\right)} \cdot \delta T_{11}-\delta T_{12}-\frac{S_{11}}{\left(1-L^{2}\right)} \cdot \delta T_{22} \\
& \delta S_{11 \mathrm{~L}} \approx-\frac{\left(1-S_{12} S_{21}\right)}{\left(1-L^{2}\right)} \cdot \delta s_{11} \\
& \frac{\delta S_{12 \mathrm{~L}}}{S_{12}}=\frac{\delta S_{21 \mathrm{~L}}}{S_{21}} \approx \frac{S_{22}}{\left(1-L^{2}\right)} \cdot \delta s_{11}+\frac{S_{11}}{\left(1-L^{2}\right)} \cdot \delta s_{22}
\end{aligned}
$$

(Index T for Through and L for Line). To obtain corresponding expressions for $\delta S_{21}$ and $\delta S_{22}$, index 1 is replaced by 2 and vice versa in Eqs. (50) to (53). 
Table 1. "disturbed" and ideal scattering matrices for the calibration elements.

\begin{tabular}{lll}
\hline & disturbed, nonideal & undisturbed, ideal \\
\hline Line 1 & $\overline{\mathbf{L}_{1} d}=\left(\begin{array}{cc}\delta T_{11} & L_{1}+\delta T_{12} \\
L_{1}+\delta T_{21} & \delta T_{22}\end{array}\right)$ & $\overline{\mathbf{L}_{\mathbf{1}}}=\left(\begin{array}{cc}0 & L_{1} \\
L_{1} & 0\end{array}\right)$ \\
\hline Line 2 & $\overline{\mathbf{L}_{2}}=\left(\begin{array}{cc}\delta s_{11} & L_{2}+\delta s_{12} \\
L_{2}+\delta s_{21} & \delta s_{22}\end{array}\right)$ & $\overline{\mathbf{L}_{2}}=\left(\begin{array}{cc}0 & L_{2} \\
L_{2} & 0\end{array}\right)$ \\
\hline Reflect & $\overline{\mathbf{R}_{d}}=\left(\begin{array}{cc}\Gamma+\delta S_{11}^{R} & 0 \\
0 & \Gamma+\delta S_{22}^{R}\end{array}\right)$ & $\overline{\mathbf{R}}=\left(\begin{array}{cc}\Gamma & 0 \\
0 & \Gamma\end{array}\right)$ \\
\hline
\end{tabular}

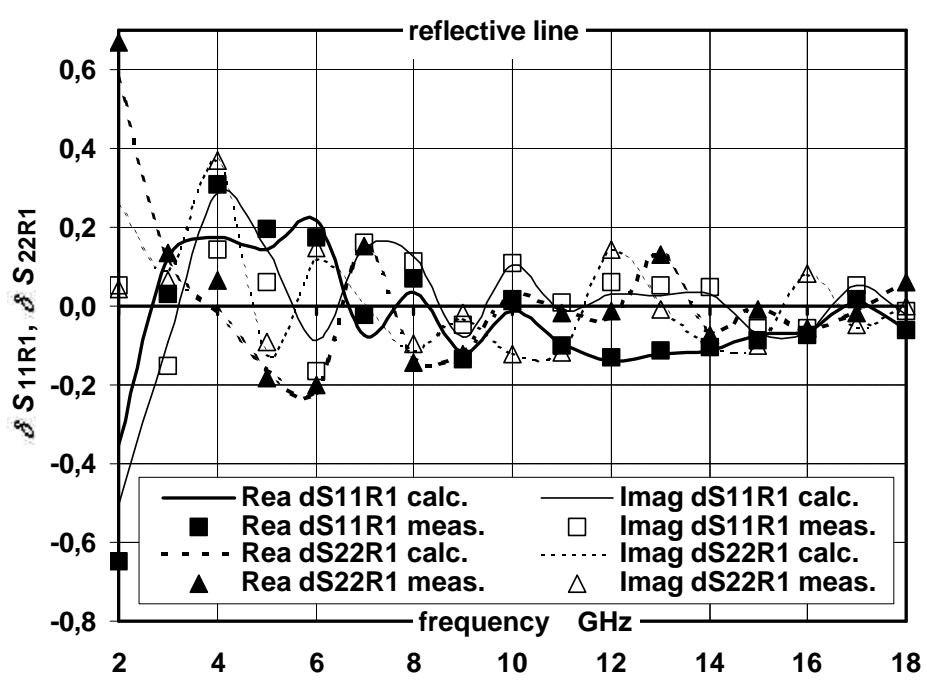

Fig. 2. Comparison of calculated and measured deviations $\left.\delta S_{11}\right|_{\mathrm{R} 1}$ and $\left.\delta S_{22}\right|_{\mathrm{R} 1} \mid$ for the high-reflective line (coaxial $\left.7 \mathrm{~mm} / \mathrm{PC}-7\right)$ as test object, using a nonideal short-circuit loaded with dielectric Teflon foils as the Reflect, instead of an ideal short-circuit on port 1 , for the TRL calibration.

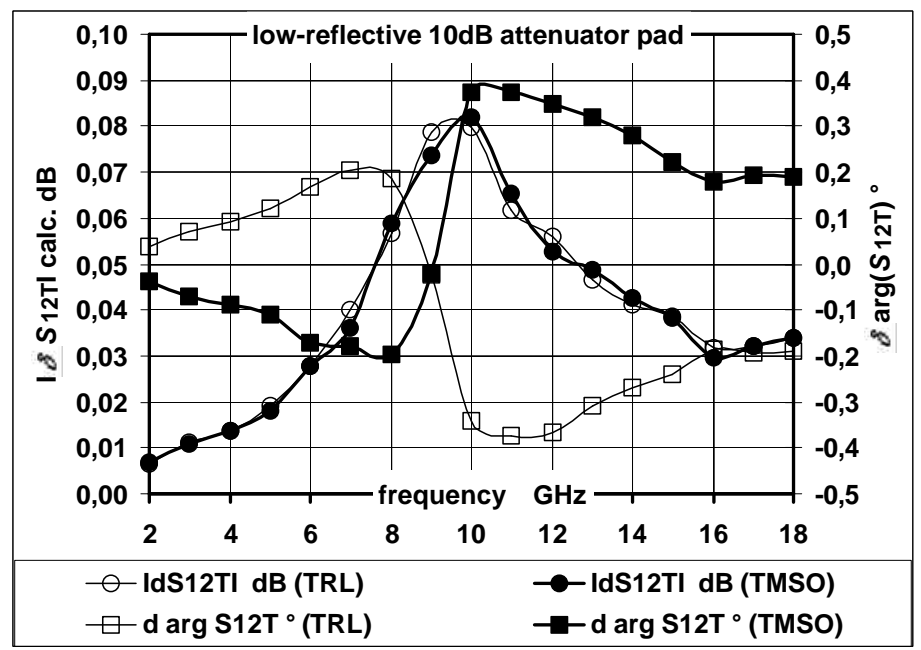

Fig. 3. Simulation: Comparison of the calculated deviations $\left|\delta S_{12 \mathrm{~T}}\right|$ in $\mathrm{dB}$ and phase deviations $\delta \arg \left(S_{12 \mathrm{~T}}\right)$ in degrees for a low-reflective $10 \mathrm{~dB}$ attenuator pad, using a nonideal Through with an $0.1 \mathrm{~mm}$ thick hair (cf. Fig. 22 of Stumper, 2003b) whose S-parameters are shown in Fig. 5 of (Stumper, 2003c), instead of an ideal Through, for the TRL and the TMSO calibration (cf. Fig. 6 of Stumper, $2003 \mathrm{c}$ ). 


\section{Discussion and experimental results}

As regards the Through and Lines, the sensitivity coefficients (Eqs. (46) to (49)) are free of all error terms but the "match" term $e_{11}$ (similar to a nonideal TMSO calibration, cf. Stumper, 2003a). They may become large if $M^{2}$ approaches 1 , e.g. if the difference $l_{2}-l_{1}$ in length of the lines approaches $\lambda / 2$, where $\lambda$ is the wavelength, (or in case of TRL, if $L^{2}$ approaches 1, e.g. if the length $l$ of the line approaches $\lambda / 2$ ). For lines taken from commercial calibration kits, this should never be the case. For example, for a line of $l=6.95 \mathrm{~mm}$ taken from a commercial $7 \mathrm{~mm} / \mathrm{PC}-7$ calibration kit, $1 /\left|1-L^{2}\right| \leq 1.74$ in the specified frequency range (2-18 GHz). Considering Eqs. (46) to (49), all deviations $\delta \mathrm{S}_{11}, \delta \mathrm{S}_{22}$ become large when using loads of small $\Gamma$ for the Reflects, while all transmission deviations are not affected by $\Gamma$, therefore short (or open) circuits should be taken. Further on we observe a term in Eqn. (47) for $\delta r_{12 \mathrm{~L} 1}$ and in Eqn. (52) for $\delta S_{12 \mathrm{~T}}$ (and also for $\delta S_{21 \mathrm{~T}}$ ) showing a direct dependence, unaffected by any other parameter, on the through transmission parameter deviations $\delta T_{12}$ (and $\delta T_{21}$, respectively), similar to a nonideal TMSO calibration (Stumper, 2003a).

The exact Eqs. (45) to (49) have been experimentally verified for the TRL case $\left(L_{1}=1\right)$ with a set of $7 \mathrm{~mm}$ coaxial two-port test objects fitted with PC-7 connectors, i.e. high-reflective devices including a step attenuator of (nominal) attenuation $0 \mathrm{~dB}, 10 \mathrm{~dB}$, and $30 \mathrm{~dB}$ and a short coaxial line, each sandwiched between the side arms of two Tjunctions, and two low-reflective attenuator pads of (nominal) attenuation $20 \mathrm{~dB}$ and $50 \mathrm{~dB}$, similar to the set described in (Stumper, 2003a). A 8510B type VNA was used for the verification. The $S_{\mathrm{jk}}$ values of the set of test objects experimentally obtained after an "ideal" TRL calibration were used in all calculations. The S-parameters of the high-reflective line varied between approximately -0.8 and +0.8 in the frequency range $2-18 \mathrm{GHz}$. Only one of the elements Through, Line, and Reflect at a time was considered nonideal.

In an example, a nonideal short-circuit at port 1 was realized by loading the "ideal" short by three dielectric Teflon foils (each of $5 \mathrm{~mm}$ diameter and $0.019 \mathrm{~mm}$ in thickness) at the connection of the inner conductor to the measuring port. The difference of the reflections of the loaded and the ideal short, both measured after an "ideal" TRL calibration, was taken as $\delta \Gamma=\delta S_{11}^{R}$ to calculate the deviations $\delta \mathrm{S}_{11 \mid R 1}$ and $\delta \mathrm{S}_{22 \mid R 1}$, for the high-reflective length of coaxial line as test object. In Fig. 2, the deviations are compared with the difference of the $S_{\mathrm{jk}}$ values obtained experimentally after TRL calibrations using either this nonideal Reflect or the ideal Reflect without foils. A second example is given in Fig. 1 of Stumper (2004) with the high-reflective $0 \mathrm{~dB}$ attenuator as test object showing the calculated deviations $\delta S_{11 \mathrm{~T}}$ and $\delta S_{12 \mathrm{~T}}$, as functions of the deviations of the actual S-parameters of a nonideal Through. The Through connection was loaded by a thin copper foil (thickness $0.03 \mathrm{~mm}$, diameter $3.2 \mathrm{~mm}$ ) introduced between the inner conductors of port 1 and 2. The difference of the reflections of the loaded and the ideal Through, both measured after an "ideal" TRL calibration, was taken to calculate the deviations $\delta S_{11 \mathrm{~T}}$ and $\delta S_{12 \mathrm{~T}}$. These are compared with the difference of the $S_{\mathrm{jk}}$ values obtained experimentally after TRL calibrations using either this nonideal Through or an "ideal" Through without foil. A third example is given in Fig. 2 of (Stumper, 2005 ${ }^{1}$ ) with the high-reflective line as test object showing the calculated and measured deviations $\delta S_{11 \mathrm{~L}}$ and $\delta S_{12 \mathrm{~L}}$. Here, for the nonideal line, the inner conductor of the line (ideal diameter $3.040 \mathrm{~mm}$ ) was replaced by an inner conductor of the same length but of diameter $3.248 \mathrm{~mm}$. For all examples, the calculated deviations $\delta S_{11}, \delta S_{12}, \delta S_{21}$, and $\delta S_{22}$ agreed well with the differences of the measured values of $S_{11}, S_{12}, S_{21}$, and $S_{22}$, respectively.

Some additional calculations were carried out to show the influence of rather small values of $\delta \Gamma, \delta T_{\mathrm{jk}}$, or $\delta s_{\mathrm{jk}}$ as they may occur in the real world during the calibration or with calibration items from commercial calibration kits. For a Line taken from a precision calibration kit, the diameters of the conductors will deviate not more than some $\mu \mathrm{m}$ from the ideal values. For $\Delta \mathrm{D}=2 \mu \mathrm{m}$, the effect on $\delta S_{12 \mathrm{~L}}$ for the high-reflective line is already considerably large. Even with this high Line precision, the deviation $\left|\delta S_{12 \mathrm{~L}}\right|$ in attenuation could be close to $0.01 \mathrm{~dB}$ for high-reflective test objects, whereas, for example, the deviations of the S-parameters of the low-reflective $20 \mathrm{~dB}$ attenuator are negligibly small.

Small particles (e.g. lints) which get in between the end planes of the connectors with the Line or Through connections, may cause considerable deviations of the S-parameters of high-reflective but also low-reflective test objects. For example, a human hair, $0.1 \mathrm{~mm}$ thick, will cause S-parameter deviations of the Through connection of about maximum 0.01 (cf. Fig. 5 in Stumper, 2003c). The deviation $\delta S_{12 \mathrm{~T}}$ (modulus and phase) for a low-reflective $10 \mathrm{~dB}$ attenuator is shown in Fig. 3. Connector imperfections such as gaps at the inner conductors may also give rise to deviations.

For all test objects, the deviation in reflection phase is equal to half the difference in phase shift of the Reflects. With a difference of $0.02 \mathrm{~mm}$ of the offset length of the short circuits, the deviation would be $0.43^{\circ}$ at $18 \mathrm{GHz}$. Only for high-reflective test objects, the reflection magnitude is influenced by the differences in phase and loss of the reflection coefficients of the Reflects. A loss difference with the Reflects has no influence on the reflection phase of the test objects.

\section{Conclusion}

Analytical expressions (sensitivity coefficients) have been developed showing the deviations of the S-parameters of test objects which depend on the deviations of the S-parameters associated with the Through, Lines, and Reflects from the ideal values which are used by the VNA firmware with the LRL and TRL calibration methods. If the reflection of the test objects is not too high, simplified expressions can be set up. Calculations and experiments show that connector imperfections such as gaps at the inner conductors and small 
particles which get in between the end planes of the connectors with the Line or Through connections or small deviations (some $\mu \mathrm{m}$ ) of the cross dimensions of the Lines from the ideal values may lead to considerable deviations and consequently contribute significantly to the uncertainty of S-parameter measurements.

Acknowledgements. The author is indebted to W. Peinelt and T. Schrader for performing the precise verification measurements.

\section{References}

Engen, G. F. and Hoer, C. A.: Thru-Reflect-Line: An Improved Technique for Calibrating the Dual Six-port Automatic Network Analyzer, IEEE Trans. Microwave Theory Tech., Vol. MTT-27, No. 12, 987-993, 1979.

Gronau, G.: Höchstfrequenztechnik, Berlin, Springer, Ch. 8, 389398, 2001.

Guidelines on the Evaluation of Vector Network Analysers (VNA), European Co-operation for Accreditation, Doc. EA-10/12, 2000.

GUM (Guide to the Expression of Uncertainty in Measurement), first Edition 1993, International Organization for Standardization (Geneva, Switzerland), corrected and reprinted, 1995.
Rytting, D.: Network Analyzer Error Models and Calibration Methods, ARFTG/NIST Short Course on RF Measurements for a Wireless World, Nov. 29-30 2001, San Diego, CA, 2001.

Stumper, U.: Influence of TMSO Calibration Standards Uncertainties on VNA S-Parameter Measurements, IEEE Trans. Instrum. Meas., vol. 52, No. 2, 311-315, 2003a.

Stumper, U.: Messabweichungen von Streuparametern nach TMSO-Kalibrierung eines Netzwerkanalysators, in: Fortschritte bei der Messung elektrischer Größen - Neue Messverfahren von DC\&LF bis HF, PTB-Bericht E-82 (ISBN 3-86509-027-3), Braunschweig, 29-62, 2003b.

Stumper, U.: Uncertainty of VNA S-Parameter Measurement Due to Non-Ideal TMSO or LMSO Calibration Standards, Advances in Radio Science (Kleinheubacher Berichte), Vol. 1, 1-8, 2003c.

Stumper, U.: Influence of Non-Ideal TRL Calibration Items on VNA S-Parameter Measurements, CPEM'2004 Digest, 2004 Conference on Precision Electromagnetic Measurements, London, 109-110, 2004.

Stumper, U.: Uncertainty of VNA S-Parameter Measurement Due to Non-Ideal TRL Calibration Items, IEEE Trans. Instrum. Meas., vol. 54, No. 2, accepted, 2005. 